\title{
Combined iNO and endothelial progenitor cells improve lung alveolar and vascular structure in neonatal rats exposed to prolonged hyperoxia
}

\author{
Aizhen Lu' ${ }^{1}$ Bo Sun ${ }^{2}$ and Liling Qian ${ }^{1}$
}

BACKGROUND: Stem cells or inhaled nitric oxide (iNO) are reported to improve lung structures in bronchopulmonary dysplasia (BPD) models. We hypothesized that combined iNO and transplanted endothelial progenitor cells (EPCS) might restore lung structure in rats after neonatal hyperoxia.

METHODS: Litters were separated into eight groups: room air, hyperoxia, hyperoxia + iNO, hyperoxia + iNO + L-NAME, hyperoxia + EPCs, hyperoxia + EPCs + L-NAME, hyperoxia + EPCs + iNO, and hyperoxia + EPCS + iNO + L-NAME. Litters were exposed to hyperoxia from the 21st day, then, sacrificed. EPCs were injected on the 21st day. L-NAME was injected daily for $7 \mathrm{~d}$ from the 21st day. Serum vascular endothelial growth factor (VEGF), radial alveolar count (RAC), VIII factor, EPCs engraftment, lung VEGF, VEGFR2, endothelial nitric oxide (eNOS) and SDF-1 expression, and NO production were examined.

RESULTS: Hyperoxia exposure led to air space enlargement, loss of lung capillaries, and low expression of VEGF and eNOS. Transplanted EPCs, when combined with iNO, had significantly increased engraftment in lungs, compared to EPCs alone, upon hyperoxia exposure. There was improvement in alveolarization, microvessel density, and upregulation of VEGF and eNOS proteins in the hyperoxia-exposed EPCs with iNO group, compared to hyperoxia alone.

CONCLUSION: Combined EPCS and iNO improved lung structures after neonatal hyperoxia. This was associated with the upregulation of VEGF and eNOS expression.

B ronchopulmonary dysplasia (BPD) is a chronic lung disease associated with significant mortality and morbidity in premature infants. New BPD is characterized by arrested alveolar and vascular growth (vascular hypothesis of BPD) $(1,2)$. Alveolar formation is a highly coordinated process between the development of airways and pulmonary vasculature (3). Exposure to inflammation caused by excessive $\mathrm{O}_{2}$ supplementation is hypothesized to interfere with the coordinated process of normal human lung development. Neonatal hyperoxia exposure is known to disrupt alveolar formation and growth, providing a useful model for studying BPD (4).

Cell-based therapy is a novel approach that offers much promise in the prevention and treatment of $\mathrm{BPD}$, including embryonic stem cells, mesenchymal stem cells, placental stem cells, umbilical cord stem cells, amniotic fluid and amnionderived cells, and lung progenitor cells. Endothelial progenitor cells (EPCs) are precursors of endothelial cells, which have the capacity of self-renewal and proliferation. EPCs is one of the key cells mediating vascular growth, which can mobilize from bone marrow and home to the site of vascular injuries, promoting neovascularization (5). There is data showing that reduced numbers or dysfunction of EPCs at birth is associated with the development of BPD $(6,7)$. In addition, a reduction in the number of EPCs in the bone marrow, circulation and lungs has also emerged in animal models of BPD (8). Evidences that angiogenic cells treatment can restore lung structure have been demonstrated. Balasubramaniam et al. (9) showed that bone marrow-derived angiogenic cells injected into the pulmonary circulation can restore lung alveolar and vascular structure after neonatal hyperoxia in infant mice. Grisafi et al. (10) showed that amniotic fluid stem cells delivered intratracheally can restore partly alveolarization and angiogenesis in an adult rat model of hyperoxia lung injury. Although stem cell therapy is a promising novel therapy for vascular injury-related diseases, studies have demonstrated that only a small portion of transplanted cells can home to the target $(11,12)$.

Previous studies suggest that impaired nitric oxide (NO) production may contribute to the pathogenesis of $\mathrm{BPD}$ $(13,14)$, and that administration of NO (inhaled NO or NO donor) can improve lung vascular and airspace structure in newborns or BPD models (15-17). However, the reasons that NO improves lung structures are not well defined, a likely hypothesis is that NO promotes angiogenesis (18). This effect is partly mediated via the synthesis of vascular endothelial growth factor (VEGF) (19). In addition, mice deficient in endothelial nitric oxide synthase (eNOS) have been shown to display reduced EPCs mobilization and impaired angiogenesis (20), suggesting that NO may be required for 
a
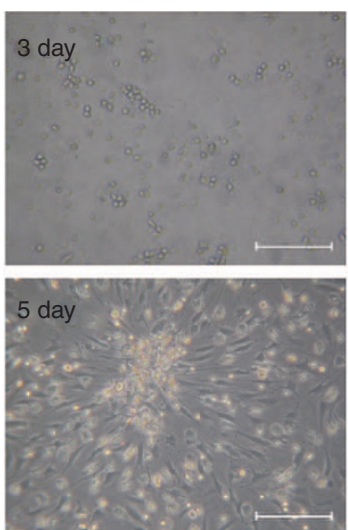

b
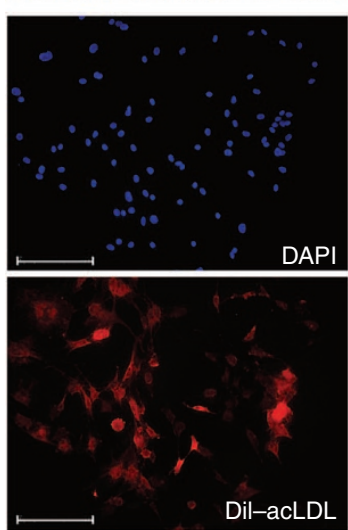

C

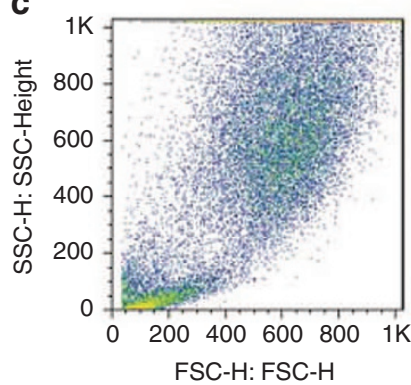

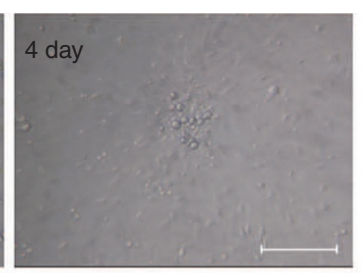
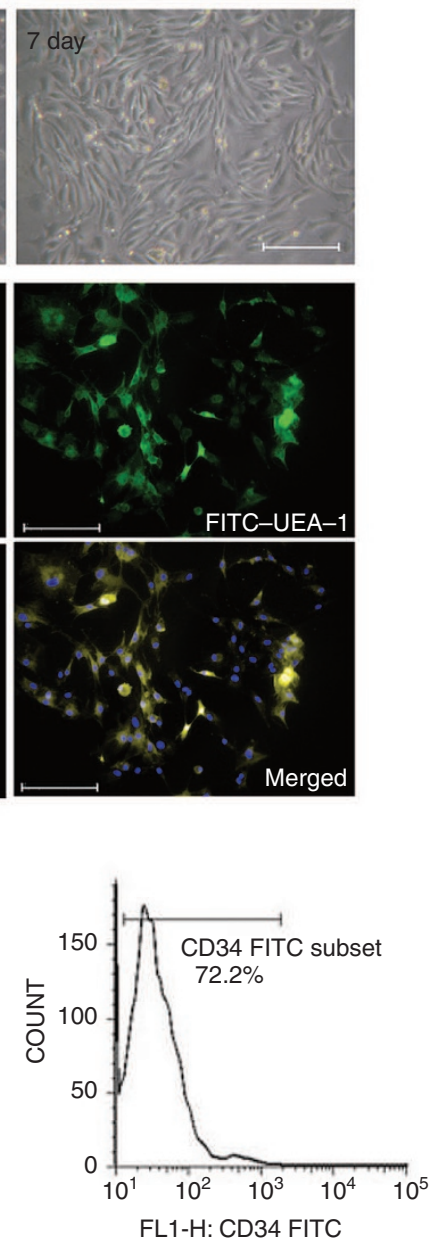

Figure 1. Characterization of isolated endothelial progenitor cells (EPCs). (a) Bone marrow derived mononuclear cells appeared as spindle-shaped and cluster arrangement after $5 \mathrm{~d}$ of culture under endothelial-specific conditions. Magnification $\times 100$, bar $=100 \mu \mathrm{m}$. (b) Testing of Dil-acLDL uptake and FITC-UEA-1 binding revealed that most of the cells were double Dil-acLDL/FITC-UEA-1 positive, indicating that the isolated mononuclear cells were EPCs. Magnification $\times 200$, bars $=50 \mu \mathrm{m}$. (c) Flow cytometry showed that the cultured mononuclear cells were mostly CD $34^{+}$cells. Left panel showed the scatter plot and right panel showed the histogram of cultured cells.

EPC-induced angiogenesis. NO has been reported to modify differentiation, survival, and adhesion of EPCs in vitro (21), which are important factors in EPCs homing to and incorporation into regions of vascular injury.

In our previous studies, we observed that circulating EPCs levels were reduced in infants with $\mathrm{BPD}$, and that inhaled NO (iNO) increased circulating EPCs number and expression of serum VEGF (22). We also found that iNO upregulated VEGF, vascular endothelial growth factor receptor 2 (VEGFR2), and

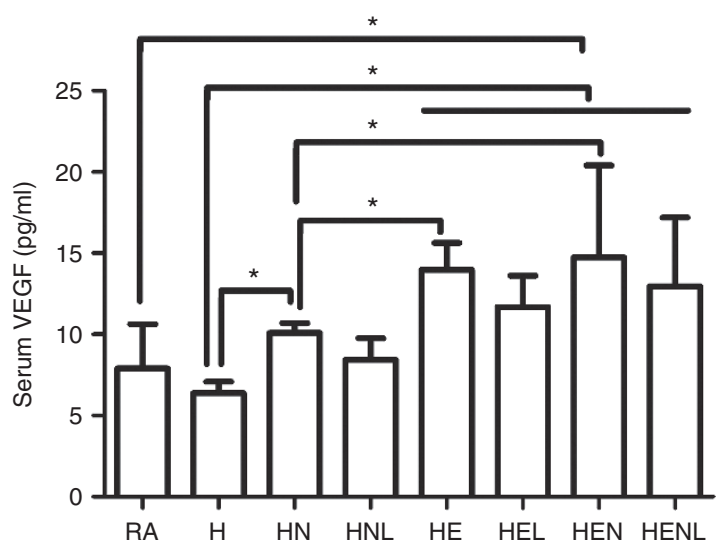

Figure 2. Effect of transplanted endothelial progenitor cells (EPCs) and inhaled nitric oxide (iNO) treatment on serum VEGF $\left(n=15,{ }^{*} P<0.05\right)$. - represents group of HE, HEL, HEN, and HENL. $\mathrm{H}$, group of hyperoxia; $\mathrm{HN}$, group of hyperoxia + iNO; HNL, group of hyperoxia + iNO + L-NAME; $\mathrm{HE}$, group of hyperoxia +EPCs; HEL, group of hyperoxia + EPCs + L-NAME; HEN, group of hyperoxia + EPCS + iNO; HENL, group of hyper-

oxia + EPCs + iNO + L-NAME; RA, group of room air; VEGF, vascular endothelial growth factor.

eNOS expression in the lungs of animal with acute lung injury and promoted EPCs mobilization from bone marrow (23), and VEGF plays an important role in EPCs homing (24). In the present study, we established a BPD model with a prolonged hyperoxia exposure, mimicking the clinical late phase of BPD. We treated the models with iNO and EPCs, and hypothesized that the combined treatment could improve structures of alveoli and vessels, which was possibly contributed by the increase of transplanted EPCs homing to the lungs associated with the upregulation of VEGF and eNOS expression in the lungs after iNO treatment.

\section{RESULTS}

\section{Characterization of EPCs}

Bone marrow-derived mononuclear cells cultured under endothelial-specific conditions developed a spindle-shaped appearance by day 5 and a typical cell cluster by day 7 (Figure 1a). The confluence of cells was about $80 \%$ at day 7 . The differentiating EPCs were positive for Dil-ac-LDL uptake and FITC-UEA-1 binding. About $85 \%$ of the cells demonstrated expression of both these markers (Figure 1b), which is compatible with differentiating EPCs. Flow cytometry demonstrated positive staining for CD34 in about $70 \%$ of cells (Figure 1c). These data confirmed that the cultured cells were mostly EPCs.

\section{Body Weight}

There was no significant difference in body weight at the day 0 among eight groups. The body weight at the day 28 was significantly different among eight groups. In comparison with room air controls (RA group), hyperoxia (H group) caused a significant reduction in body weight $(67.5 \pm 4.4 \mathrm{~g}$ vs. $46.0 \pm 2.7 \mathrm{~g}, P<0.05)$. Body weight was higher in the animals of iNO and/or EPCs treatment groups (hyperoxia + iNO (HN), hyperoxia + iNO + L-NAME (HNL), hyperoxia +EPCs (HE), 
a
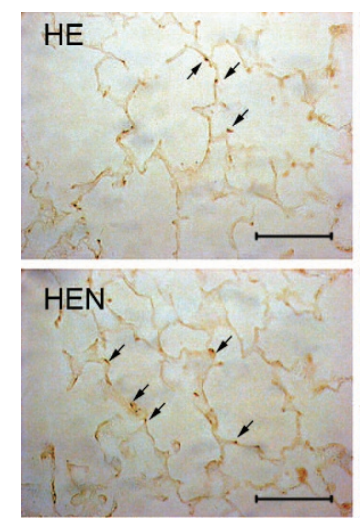

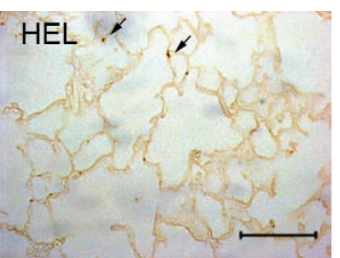

HENL
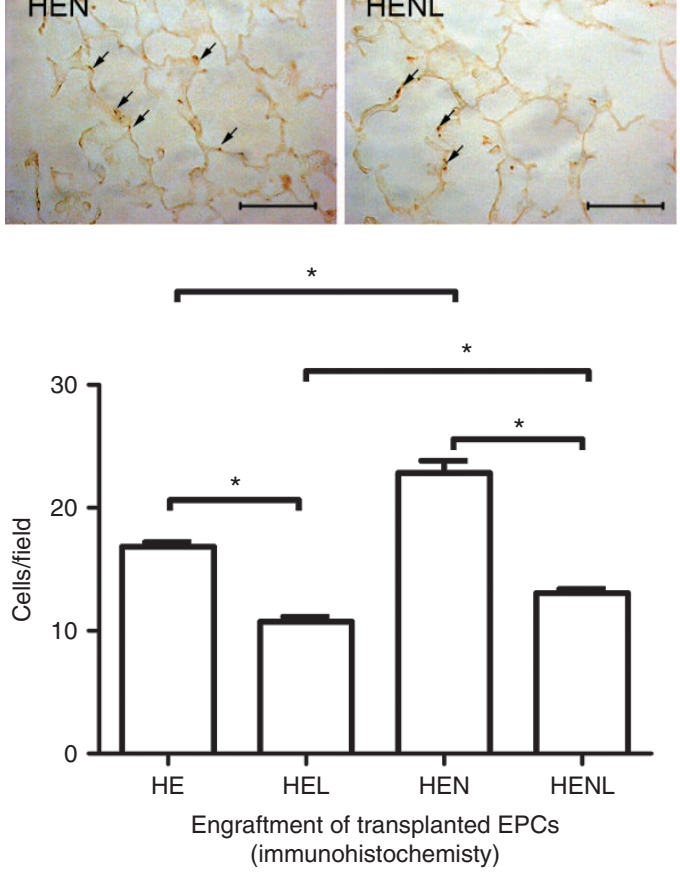
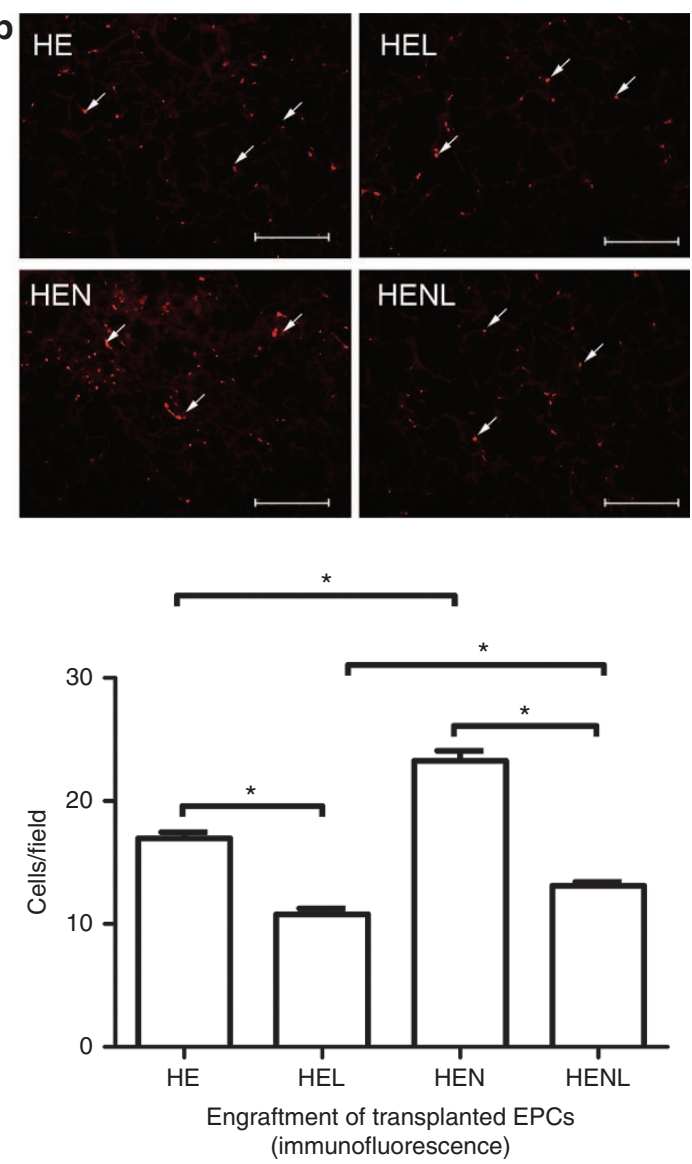

Figure 3. Engraftment of transplanted endothelial progenitor cells (EPCs) in lungs. (a) Engraftment of transplanted EPCs in lungs with immunohistochemical 5-Bromo-2-deoxyUridine (BrdU) staining. Upper panel shows the representative photomicrographs; black arrows point to the stained engrafted cells. Magnification $\times 200$, bar $=50 \mu \mathrm{m}$. The bottom panel shows the mean number of transplanted EPCs per high-powered field $\left(n=15,{ }^{*} P<0.05\right)$. $(\mathbf{b})$ Transplanted EPCs engraftment in lungs with immunofluorescent BrdU staining. Upper panel shows the representative photomicrographs; white arrows point to the stained engrafted cells. Magnification $\times 100$, bar $=100 \mu \mathrm{m}$. The bottom panel shows the mean number of transplanted EPCs per high-powered field $\left(n=15,{ }^{*} P<0.05\right)$. HE, group of hyperoxia +EPCs; HEL, group of hyperoxia + EPCs + L-NAME; HEN, group of hyperoxia + EPCs + iNO; HENL, group of hyperoxia + EPCs + iNO + L-NAME.

hyperoxia + EPCs + L-NAME (HEL), hyperoxia + EPCs + iNO $(\mathrm{HEN})$, and hyperoxia + EPCs + iNO + L-NAME (HENL)) comparing with that in mono-hyperoxia group $(\mathrm{H})$ $(50.9 \pm 1.6 \mathrm{~g}, 40.5 \pm 0.9 \mathrm{~g}, 52.2 \pm 1.8 \mathrm{~g}, 50.1 \pm 0.7 \mathrm{~g}, 50.3 \pm 3.1 \mathrm{~g}$, $0.2 \pm 1.66 \mathrm{~g}$ vs. $46.0 \pm 2$. $7 \mathrm{~g}, P<0.05$, respectively). Body weights were not significantly different among iNO and/or EPCs treatment groups.

\section{Serum VEGF}

There was a significant difference among the serum VEGF concentration of the eight groups (Figure 2). The serum VEGF in the HN group was significantly higher than that in the $H$ group $(13.9 \pm 1.66 \mathrm{pg} / \mathrm{ml}$ vs. $6.38 \pm 0.719 \mathrm{pg} / \mathrm{ml}, P<0.05, n=15)$. And the serum VEGF in the HE group was significantly higher than that in the HN group $(14.7 \pm 5.64 \mathrm{pg} / \mathrm{ml}$ vs. $13.9 \pm 1.66 \mathrm{pg} / \mathrm{ml}$, $P<0.05, n=15)$. The serum VEGF level in EPCs intervention groups (HE, HEL, HEN, and HENL groups) was significantly higher than that in the non-EPCs intervention groups (RA and $\mathrm{H}$ groups, $P<0.05$, respectively). There was no difference in concentration among EPCs intervention groups (HE, HEL, HEN, and HENL groups).

\section{Engraftment of Transplanted EPCs in Lungs}

Immunohistochemistry and immunofluorescence revealed transplanted EPCs labeled with 5-Bromo-2-deoxyUridine (BrdU) engrafted in subendothelial and alveolar interstitial locations of the lung (Figure $3, n=15$ ). iNO (HEN group) promoted greater localization of transplanted EPCs in the lungs than EPCs alone (HE group) $(P<0.05)$, treatment of neonatal rats with L-NAME (HEL and HENL group) suppressed the localization of transplanted EPCs in lungs $(P<0.05$, respectively) more than EPCs intervention alone (HE group) or EPC plus iNO (HEN group).

\section{Lung Morphometry}

Neonatal exposure to hyperoxia resulted in a simplified lung structure, characterized by enlarged air spaces and a reduction in the complexity of lung structure (Figure 4a, RA vs. H; $n=15)$. The restoration of lung air space was quantified by morphometric analysis (Figure $4 c, n=15$ ). Comparing with RA group, the radial alveolar count (RAC) in the hyperoxia groups (H, HN, HNL, HE, HEL, HEN, and HENL) were significantly less $(P<0.05$, respectively). Comparing with mono-hyperoxia 

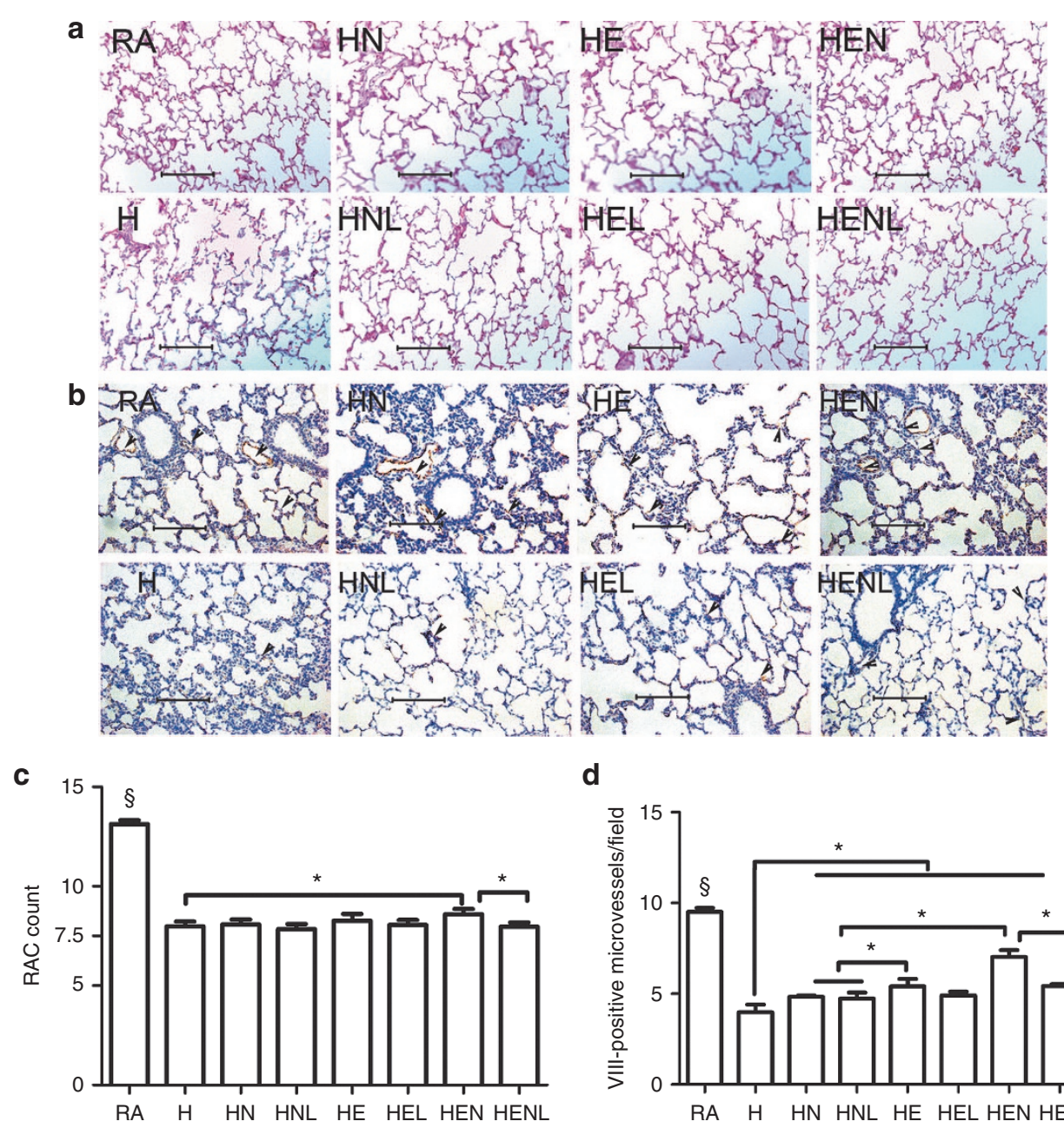

d

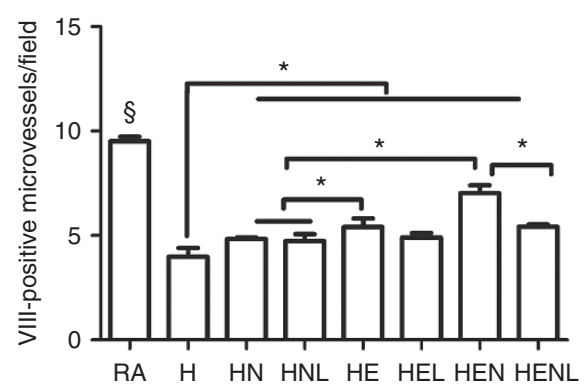

Figure 4. Effects of transplanted endothelial progenitor cells (EPCs) and inhaled nitric oxide (iNO) treatment on RAC and microvessel density. (a) Representative photomicrographs illustrating lung morphology from different groups. Magnification $\times 200$, bar $=50 \mu \mathrm{m}$. (b) Representative photomicrographs illustrating factor VIII staining used to identify microvessel density in the lungs. Black arrows point to microvessels, magnification $\times 200$, bar $=50 \mu \mathrm{m}$. (c) Effects of transplanted EPCs and iNO treatment on RAC ( $n=9$; ${ }^{5} P<0.05 \mathrm{vs}$. H, HN, HNL, HE, HEL, HEN, and HENL; $\left.{ }^{*} P<0.05\right)$. (d) Effects of transplanted EPCS and iNO treatment on microvessel density $\left(n=9 ;{ }^{\circledR} P<0.05\right.$ vs. $\mathrm{H}, \mathrm{HN}, \mathrm{HNL}, \mathrm{HE}, \mathrm{HEL}, \mathrm{HEN}$, and HENL; $\left.{ }^{*} P<0.05\right)$. - represents group of $\mathrm{HN}, \mathrm{HNL}, \mathrm{HE}, \mathrm{HEL}, \mathrm{HEN}$, and HENL. H, group of hyperoxia; HN, group of hyperoxia + iNO; HNL, group of hyperoxia + iNO + L-NAME; HE, group of hyperoxia $+E P C s ; \mathrm{HEL}$, group of hyperoxia + EPCs + L-NAME; HEN, group of hyperoxia + EPCs + iNO; HENL, group of hyperoxia + EPCs + iNO + L-NAME; RA, group of room air; RAC, radial alveolar count.

treatment $(\mathrm{H})$, treatment with iNO (HN) or EPCs (HE) only did not increase RAC significantly $(8.03 \pm 0.27,8.26 \pm 0.34$ vs. $7.98 \pm 0.23$, respectively). However, combined iNO and EPC treatment improved the RAC significantly compared with hyperoxia treatment $(8.59 \pm 0.26$ vs. $7.98 \pm 0.23, P<0.05)$.

\section{Lung Microvessels}

The lung microvessel density was significantly different among eight groups (Figure $4 \mathbf{b}, \mathbf{d}, n=15$ ). Factor VIII staining showed that the lung microvessel density of hyperoxic rats $(\mathrm{H}, \mathrm{HN}$, HNL, HE, HEL, HEN, and HENL) was significantly less than that of normoxic rats (RA) $(P<0.05$, respectively). iNO and/ or EPCs treatments (HN, HNL, HE, HEL, HEN, and HENL) increased microvessel density significantly compared with hyperoxia alone ( $\mathrm{H}$ group) $(P<0.05$, respectively). Comparing with iNO treatment (HN and HNL), EPCs treatment (HE and HEN) increased microvessel density dramatically $(P<0.05$, respectively). L-NAME (HENL group) significantly inhibited the improvement of microvessel density compared with EPCs plus iNO intervention $(\mathrm{HEN})(P<0.05)$.

\section{Lung VEGF, VEGFR2, and eNOS Expression}

There was a significant difference in VEGF mRNA expression among the eight groups (Figure $5 \mathrm{a}, n=15$ ). VEGF mRNA expression was significantly lower in the hyperoxia groups $(\mathrm{H}$, HN, HNL, HE, HEL, HEN, and HENL) than that in the RA group $(P<0.05$, respectively). EPCs treatment (HE) improved VEGF mRNA expression significantly than that in the $\mathrm{H}$ group $(0.69 \pm 0.09$ vs. $0.23 \pm 0.16, P<0.05)$. L-NAME (HEL) inhibited this increase $(0.31 \pm 0.08$ vs. $0.69 \pm 0.09, P<0.05)$. There was a significant difference in VEGF protein expression among the eight groups (Figure $5 \mathbf{b}, n=15)$. The hyperoxia group $(\mathrm{H})$ had less VEGF protein expression than that in the RA group $(0.52 \pm 0.01$ vs. $0.82 \pm 0.01, P<0.05)$. iNO $(\mathrm{HN})$ or EPCs treatment $(\mathrm{HE})$ 

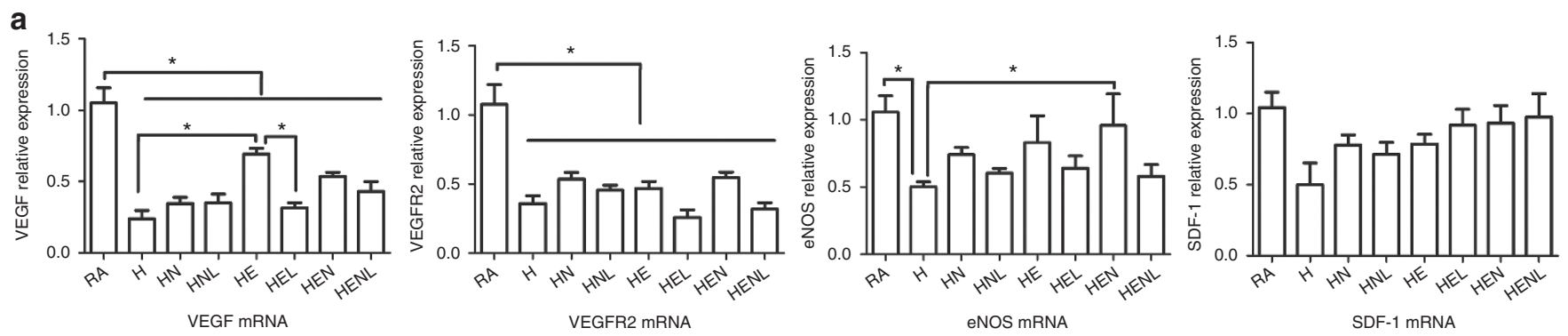

b
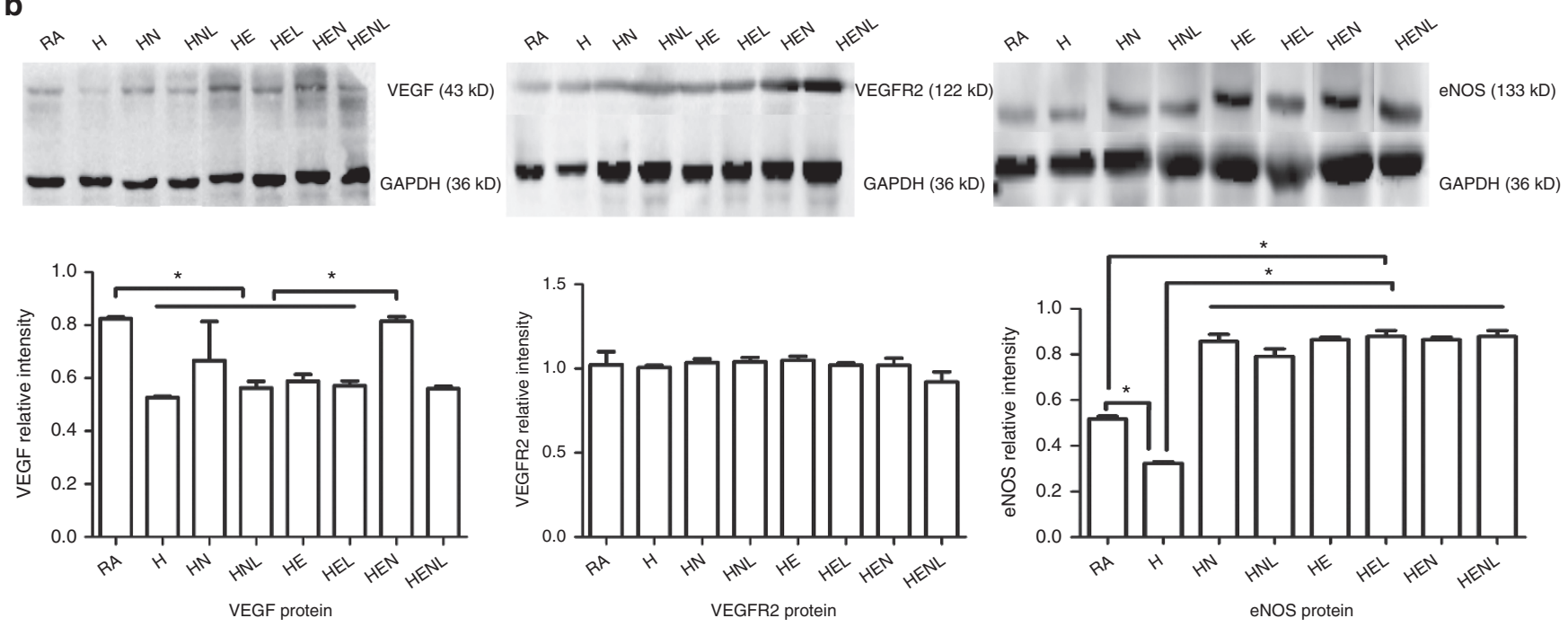

Figure 5. Effect of transplanted endothelial progenitor cells (EPCs) and inhaled nitric oxide (iNO) treatment on lung VEGF, VEGFR2, eNOS, and SDF-1 expression. (a) Effect of transplanted EPCs and iNO treatment on mRNA expression of lung VEGF, VEGFR2, eNOS, and SDF-1 ( $n=15$, * $P<0.05)$-in the first and second panel represents groups of $\mathrm{H}, \mathrm{HN}, \mathrm{HNL}, \mathrm{HE}, \mathrm{HEL}, \mathrm{HEN}$, and HENL. (b) Effect of transplanted EPCs and iNO treatment on protein expression of lung VEGF, VEGFR2, and eNOS. The upper panel shows the protein expression of lung VEGF, VEGFR2, and eNOS by western blot, and the bottom panel shows the effects of EPCs and iNO treatment on protein expression of lung VEGF, VEGFR2, and eNOS $\left(n=15,{ }^{*} P<0.05\right)$-in the subpanel of VEGF relative intensity represents groups of $\mathrm{H}, \mathrm{HN}, \mathrm{HNL}, \mathrm{HE}$, and $\mathrm{HEL}-$ in the subpanel of eNOS relative intensity represents HN, HNL, HE, HEL, HEN, and HENL. eNOS, endothelial nitric oxide synthase; $\mathrm{H}$, group of hyperoxia; $\mathrm{HN}$, group of hyperoxia $+\mathrm{iNO}$; $\mathrm{HNL}$, group of hyperoxia $+\mathrm{iNO}+\mathrm{L}-\mathrm{NAME} ; \mathrm{HE}$, group of hyperoxia $+E P C s ; H E L$, group of hyperoxia + EPCs + L-NAME; HEN, group of hyperoxia + EPCs + iNO; HENL, group of hyperoxia + EPCs + iNO + L-NAME; RA, group of room air; SDF-1, stromal cell-derived factor-1; VEGF, vascular endothelial growth factor; VEGFR2, vascular endothelial growth factorreceptor 2. GAPDH, glyceraldehyde-3-phosphate dehydrogenase.

only did not increase VEGF protein expression in the lung. The EPC plus iNO (HEN) improved VEGF protein expression than iNO (HN) or EPCs (HE) did $(P<0.05$, respectively).

There was a significant difference in VEGFR2 mRNA expression among the eight groups (Figure $5 \mathrm{a}, n=15$ ). VEGFR2 mRNA expression was significantly lower in the hyperoxia intervention groups $(\mathrm{H}, \mathrm{HN}, \mathrm{HNL}, \mathrm{HE}, \mathrm{HEL}, \mathrm{HEN}$, and HENL) than in the air group $(P<0.05$, respectively). However, no significant difference was found in VEGFR2 protein expression among the six groups (Figure $5 \mathbf{b}, n=15$ ).

There was a significant difference in the eNOS mRNA expression among the eight groups (Figure $5 \mathrm{a}, n=15$ ). The hyperoxia group $(\mathrm{H})$ had significantly less eNOS mRNA expression than that in the RA group $(0.46 \pm 0.10$ vs. $0.05 \pm 0.36, P<0.05)$. iNO (HN) or EPCs treatment (HE) only did not increase eNOS expression in the lung. The EPCs plus iNO improved eNOS mRNA expression than hyperoxia $(\mathrm{H})(0.96 \pm 0.57$ vs. $0.46 \pm 0.10, P<0.05)$. There was a significant difference in eNOS protein expression among the eight groups (Figure $5 \mathbf{b}$, $n=15)$. Compared with the RA group, hyperoxia $(\mathrm{H})$ inhibited the eNOS protein expression significantly $(0.32 \pm 0.01$ vs. $0.51 \pm 0.03, P<0.05)$. eNOS protein expression was significantly higher in the iNO and/or EPCs treatment groups (HN, HNL, HE, HEL, HEN, and HENL) than that in the RA or $\mathrm{H}$ group $(P<0.05$, respectively).

No difference was found in the SDF-1 mRNA expression among the eight groups (Figure 5a).

\section{NO Production}

There was no significant difference in lung NO production among the eight groups.

\section{DISCUSSION}

We found that ex vivo generated EPCs could home to hyperoxic lungs of neonatal rats. iNO improved the engraftment of transplanted EPCs in hyperoxic lungs. Combined EPC transplantation and iNO could improve lung structures after neonatal hyperoxia. This may be associated with the upregulation of VEGF and eNOS expression in the lungs. 


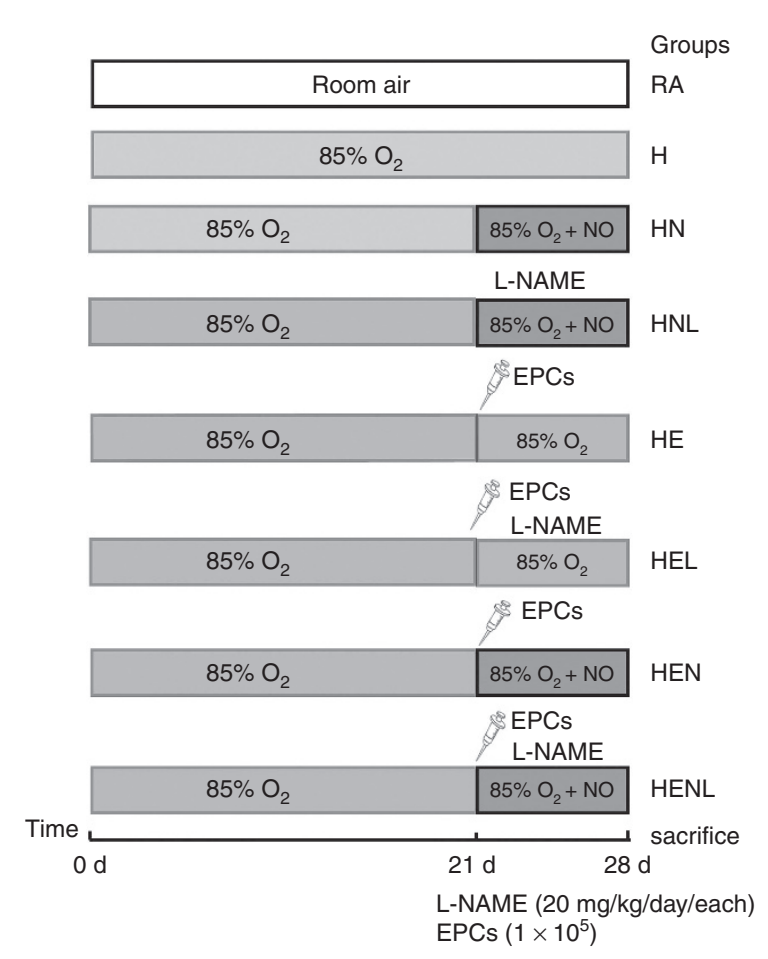

Figure 6. Study design and treatment protocols. One-day-old Sprague-Dawley rats were maintained in room air (group of RA) or exposed to hyperoxia $\left(\mathrm{FiO}_{2} 85 \%\right.$, group of $\mathrm{H}, \mathrm{HN}, \mathrm{HNL}, \mathrm{HE}, \mathrm{HEL}, \mathrm{HEN}$, and HENL). Litters had been exposed to iNO ( $5 \mathrm{ppm}$ ) for $7 \mathrm{~d}$ from the 21 st day of the experiment in the group of HN, HNL, HEN, and HENL. Animals received EPCs $\left(1 \times 10^{5}\right.$ cells) by tail vein injection on the $21 \mathrm{st}$ day of the experiment in the group of HE, HEL, HEN, and HENL. Animals were administered with L-NAME ( $20 \mathrm{mg} / \mathrm{kg} /$ day) daily for $7 \mathrm{~d}$ by intraperitoneal injections from the 21st day of the experiment in the group of HNL, HEL, and HENL. All litters were sacrificed on the 28th day of the experiment for blood and lung evaluations. EPCs, endothelial progenitor cells; $\mathrm{H}$, group of hyperoxia; $\mathrm{HN}$, group of hyperoxia $+\mathrm{iNO} ; \mathrm{HNL}$, group of hyperoxia + $\mathrm{iNO}+\mathrm{L}-\mathrm{NAME} ; \mathrm{HE}$, group of hyperoxia +EPCs; HEL, group of hyperoxia + EPCs + L-NAME; HEN, group of hyperoxia + EPCs + iNO; HENL, group of hyperoxia + EPCS + iNO + L-NAME; RA, group of room air.

\section{Identification of EPCs}

The identification of EPCs included morphological, functional, and phenotypic testing. The cultured EPCs were spindle-shaped cells. EPCs are endothelial precursor cells that have endothelial cell function. This includes ac-LDL uptake and UEA-1 binding (25). At present, there is controversial regarding EPC phenotype. Murine bone marrow-derived EPCs have a high $\mathrm{CD} 34^{+}$cell content (26). The cells we cultured were spindle-shaped, demonstrated ac-LDL uptake and UEA-1 binding, and expressed CD34. This suggested that the cells we cultured were EPCs.

\section{Combined EPC Transplantation and iNO Improved Lung Structure in an Animal Model of Hyperoxic Lung Injury}

Injected venous EPCs migrated and engrafted into lungs of neonatal hyperoxic rats, and improved lung vascularity significantly. Mono-EPCs treatment did not improve alveolar structure as determined by RAC measurements. When combined with iNO, both the lung airspaces and vascularity were significantly improved, suggesting that EPCs transplantation and iNO can effect synergistically in hyperoxic lungs.

EPCs may have a beneficial effect on BPD. The distal lung harbors EPCs (27) that contribute to lung repair, and their depletion in the developing lung is associated with arrested lung development $(28,29)$. Studies showed that provided angiogenic cells could promote alveolar and vascular growth after neonatal hyperoxic exposure, and restore lung structure to normal in infant mice (9). However, in this study, we found that in the late phase of BPD, a single dose of EPCs administered through the peripheral venous route only increased lung microvessels, with no improvement in alveolar structure as determined by RAC measurements. Compared with the two previous studies $(9,10)$, the reason why mono-EPCs treatment did not result in any improvement in alveolar structure could be due to the selected route of administration.

It has been reported that iNO can improve lung structure after hyperoxic injury. Inflammation plays an important role in the development of hyperoxic lung injury. iNO can downregulate NF- $\mathrm{KB}$ transcription factors (30), reduce expression of proinflammatory cytokines, inhibit leukocyte trafficking in lungs and release of inflammatory mediators (31), protect barrier function of the alveolar capillary membrane (32), and increase the antioxidant capacity of the lungs (33). In addition, provided with NO donor (17), could improve alveolar and vascular growth of hyperoxia-injuried lungs, which may be related with the increased expression of VEGF and eNOS in the lung. In this study, we used iNO from the 21st day of the experiment with a low dose of $5 \mathrm{ppm}$. This mono-iNO treatment did not improve neither vascular nor alveolar growth, and this may be related to the dose of iNO used. However, we found that mono-iNO treatment improved serum and lung VEGF expression. We also found that addition of iNO could increase the engraftment of EPCs in lungs, increase lung microvessels and improve alveolar structure.

\section{Lung eNOS and VEGF Were Increased When Combining EPCs Transplantation With iNO}

Hyperoxia decreased the amount of lung VEGF and eNOS expression. iNO and/or EPCs treatment increased VEGF mRNA and eNOS expression in the lungs. Compared with iNO or EPCs alone, EPCs plus iNO promoted more transplanted EPCs engraftment in the lungs and increased VEGF protein expression, but did not increase eNOS expression in the lungs.

eNOS is a biomarker for EPCs (34). In vitro studies have shown that eNOS/NO signaling enhance the mobilization, homing, and adhesion of EPCs $(35,36)$. eNOS/NO signaling is associated with BPD (13). On the basis of these studies, we speculated that transplanted EPCs engrafted the lungs, expressed more eNOS, produced more internal NO, and enhanced production of VEGF mRNA in the lungs.

EPCs plus iNO increased VEGF expression in the lungs. VEGF is an important growth factor for angiogenesis during lung development. It was reported that NO induces VEGF synthesis (37), and VEGF induces EPCs mobilization and migration and incorporation into damaged vessels (20). We 
speculated that iNO increased NO levels in the lungs, stimulating local VEGF production, which helped capture migrating EPCs for new lung vasculature. However, we found no significant difference in lung NO production in the eight groups.

SDF-1 is an important cytokine for EPCs mobilization and homing. However, in our study, we found that hyperoxia decreased the expression of SDF-1, but it was not statistically significant. Transplanted EPCs and EPCs plus iNO did not improve SDF-1 expression in the lungs. Qi et al. (23) reported that the expression of SDF-1 was elevated in plasma from a piglet model of acute lung injury. There are few reports of SDF-1 expression in lung diseases. We speculate that variable detection times may be the reason for these inconsistent results.

The present study has some limitations. First, we did not test different doses of iNO to find the optimal concentration of improving lung structure. Second, we used L-NAME (a panNOS inhibitor) to suppress the activity of eNOS. However, L-NAME also can decrease matrix metalloproteinase-12 (MMP-12) and increase in matrix metalloproteinase inhibitor 1 (TIMP-1) and TIMP-2 expression, leading to improved alveolar structure (38). We did not examine this effect in this study.

Collectively, our animal model mimics one extreme clinical situation, namely those newborns who need oxygen for a very long period even if their lung growth has already arrested. Our study showed the therapeutic effects of combining EPCs transplantation with iNO on the arrested lung growth of long period hyperoxic injury. It has been reported that EPCs improve lung structure by secreting angiocrine, which induces and sustains regenerative lung alveolarization (39). In addition, NO has been reported to promote angiogenesis (18). In this study, we observed that lung eNOS and VEGF expression were increased after this combining treatment, suggesting that combining iNO and EPCs promote angiogenisis synergistically. However, the exact mechanism of how combination treatment improves lung structure is a more complicated process, requiring further investigation.

\section{METHODS}

\section{Ethics Statement}

Animal study protocols were carried out in conformity with the Institutional Guidelines for the Care and Use of Laboratory Animals and approved by the Animal Ethics Committee of Children's Hospital of Fudan University (Permit Number: 2010002). All surgery was performed under anesthesia, and all efforts were made to minimize suffering.

\section{Animals and Study Design}

Four-week-old Sprague-Dawley rats weighing 60-80 g and 18-d pregnant Sprague-Dawley rats were purchased from Shanghai Slaccas Laboratory Animal and maintained at the animal center of our hospital. Litters from pregnant rats were allowed to deliver and recover in room air for $24 \mathrm{~h}$ after birth. Neonatal rats were randomly distributed into the following eight groups: RA, H, HN, HNL, HE, HEL, HEN, and HENL. Fifteen newborn rats were placed in each group (Figure 6). Briefly, litters with hyperoxia were maintained in a chamber and exposed to $85 \% \mathrm{O}_{2}$ for $28 \mathrm{~d}$. Tail vein injection of EPCs $\left(1 \times 10^{5}\right.$ cells) was performed on the 21 st day of the experiment in the group of HE, HEL, HEN, and HENL. L-NAME was injected intraperitoneally daily for $7 \mathrm{~d}(20 \mathrm{mg} / \mathrm{kg} /$ day $)$ from the 21st day of the experiment in the group of HNL, HEL, and HENL. iNO (5 ppm) has been administered for $7 \mathrm{~d}$ from the 21 st day of the experiment in the group of
HN, HNL, and HENL. NO gas at 1,000 ppm (Noventek, Shanghai, China) was supplied to the inspiratory line, and mixed gas containing $85 \% \mathrm{O}_{2}$ and $\mathrm{NO}$ was delivered to the chamber at a flow rate of $9-10$ $1 / \mathrm{min}$. This rapid flow was employed to minimize the transit time of $\mathrm{NO}$ in the chamber, thereby lowering the concentration of $\mathrm{NO}_{2}$. Concentrations of $\mathrm{NO}$ and $\mathrm{NO}_{2}$ were monitored continuously at the exhalation line with an electrochemical $\mathrm{NO} / \mathrm{NO}_{2}$ analyzer (Bedfont, Rochester, UK). Measured $\mathrm{NO}_{2}$ concentration was less than $1 \mathrm{ppm}$ during experiment. Silicon dioxide was used to maintain humidity less than $70 \%$ and soda lime to maintain $\mathrm{CO}_{2}$ levels less than $0.05 \%$. Inspired oxygen levels and humidity were monitored and recorded three times daily. Lactating dams were switched daily between hyperoxia exposure and room air to minimize prolonged oxygen toxicity.

\section{Isolation and Characterization of Bone Marrow-Derived EPCs}

Mononuclear cells were isolated from the femoral and tibia bone marrow of 4-wk-old rats using density gradient centrifugation with percoll-1083 (Sigma, St. Louis, MO). Cells were placed on six-well plates coated with fibronectin (Sigma), and cultured in EGM-2MV medium (Lonza, Allendale, NJ). After 3 d culture, nonadherent cells were removed by washing with Dulbecco's phosphate-buffered saline, and then new medium was added. At the 7th to 10th day, the confluence of adherent cells was above $80 \%$ and cells were harvested for in vitro studies. Briefly, cells were first incubated with Dil-acetylated low-density lipoprotein (Dil-ac-LDL, final concentration of $10 \mu \mathrm{g} /$ $\mathrm{ml}$; Biomedical Technologie, Ward Hill, MA) at $37^{\circ} \mathrm{C}$ for $4 \mathrm{~h}$ and then treated with FITC-labeled ulex europaeus agglutinin-1 (FITCUEA-1, final concentration of $10 \mu \mathrm{g} / \mathrm{ml}$; Sigma) for $1 \mathrm{~h}$. After staining, cells were fixed with $4 \%$ paraformaldehyde for $10 \mathrm{~min}$. Samples were washed with Dulbecco's phosphate-buffered saline three times and viewed for double staining cells using a fluorescence microscope. CD34 expression on EPCs was analyzed using flow cytometry (BD FACS Cabibur).

\section{Labeling EPCs With BrdU and EPC Transplantation}

EPCs were labeled prior to injection. Briefly, $10 \mu \mathrm{l}$ of BrdU solution $(1 \mathrm{mmol} / \mathrm{l} \mathrm{BrdU}$ in $1 \times$ Dulbecco's phosphate-buffered saline; BD Bioscience, San Jose, CA) was added to each $\mathrm{ml}$ of culture media $72 \mathrm{~h}$ prior to harvesting cells. The harvested cells were then resuspended in saline to $1 \times 10^{5} / 200 \mu \mathrm{l}$, and $200 \mu \mathrm{l}$ was injected into each rat tail vein.

\section{Body Weight}

Each animal was weighted at the day 0 and day 28 of the experiment.

\section{Processing of the Lungs}

The animals were sacrificed on the 28th day. Briefly, animals were anesthetized, and thoracic cavity was opened. Blood sample was drawn from the right ventricle for serum VEGF test. Vascular blood in lungs was removed by $20 \mathrm{ml}$ of cool saline perfusion through a cannula placed into the pulmonary artery with left auricle cut. Then, the lungs and heart were removed en bloc, and the right lung was cut away in liquid nitrogen for real-time PCR (upper right lobe), western blot (middle right lobe), and NO (lower right lobe) assays. The left lung was perfused for $30 \mathrm{~min}$ via pulmonary artery with $4 \%$ paraformaldehyde at a pressure of $60 \mathrm{~cm} \mathrm{H}_{2} \mathrm{O}$ while the lungs were first inflated to an airway pressure of $30 \mathrm{~cm} \mathrm{H}_{2} \mathrm{O}$ for $1 \mathrm{~min}$, and then deflated to $10 \mathrm{~cm} \mathrm{H}_{2} \mathrm{O}$ for the rest time. The left lungs were removed and fixed for another $12 \mathrm{~h}$ in the $4 \%$ paraformaldehyde for immunohistochemical analysis.

\section{ELLISA}

The serum concentration of VEGF was quantified using a commercial ELLISA kit (Abcam, Cambridge, UK). The ELLISA was performed according to the manufacturer's instructions.

\section{Lung Morphometry}

The formalin-fixed left lungs were paraffin-embedded and cut into 5 - $\mu$ m-thick serial sections. Lung sections were stained with hematoxylin and eosin. The RAC was assessed as previously described (40).

\section{Immunohistochemistry for Factor VIII}

Lung sections were stained for the presence of factor VIII (von Willebrand factor; 1:200; Biocare, Concord, CA), an 
endothelial-specific marker. Images of factor VIII-stained slides were captured using a $\times 20$ objective and evaluated to assess microvessel density. The number of factor VIII-positive vessels was counted for each high-powered field. Images were captured randomly from five nonoverlapping high-powered fields from each slide, and three slides were examined per animal. Results were expressed as number of vessels per field.

\section{Immunohistochemistry and Immunofluorescence of Transplanted EPCs}

BrdU has been widely used to label cells since 1970s, and reports have described the migration and differentiation of BrdU-labeled cells in vivo after longer periods of time. In this study, we use immunohistochemistry and immunofluorescence to detect BrdU to make it more accurate.

Immunohistochemistry. Lung sections were incubated with $50 \%$ formamide/ 2 SSC and $2 \mathrm{~mol} / \mathrm{l} \mathrm{HCl}$ and then washed with Tris-buffered saline. Sections were incubated with BrdU antibody (1: 400 dilution; Santa Cruz Biotechnology, Dallas, TX) at $4{ }^{\circ} \mathrm{C}$ overnight, then incubated with corresponding biotinylated secondary antibodies and avidin-biotin-peroxidase (Vectastain Elite ABC kit; 1:200 dilution; Vector Laboratories, Burlingame, CA) for $45 \mathrm{~min}$ at $37^{\circ} \mathrm{C}$. Immunoreactivity was visualized using diaminobenzidine.

Immunofluorescence. Lung sections were incubated with $50 \%$ formamide $/ 2$ SSC and $2 \mathrm{~mol} / \mathrm{l} \mathrm{HCl}$ and then washed with Tris-buffered saline. Sections were then incubated with BrdU antibody (APC-BrdU antibody; 1:500 dilution; $\mathrm{BD}$ Bioscience) at $37^{\circ} \mathrm{C}$ for $2 \mathrm{~h}$, washed, and examined. Digital images were captured using a Leica digital imaging system at $20 \times$ objective with random sampling of all the tissue in an unbiased fashion for quantitative analysis. Images were captured randomly from five nonoverlapping fields from each slide. Three slides were examined per each animal.

\section{Quantitative Real-Time PCR}

Total lung RNA was extracted from frozen lung tissue using trizol reagent. One microgram of RNA was reverse-transcribed to make cDNA using the commercial RT reagent Kit (TaKaRa, Shiga, Japan). Real-time PCR was performed with $2 \mu \mathrm{l}$ of cDNA using the commercial real time PCR Kit (TaKaRa) for 40 cycles $\left(95^{\circ} \mathrm{C}\right.$ for $20 \mathrm{~s}, 58$ ${ }^{\circ} \mathrm{C}$ for $30 \mathrm{~s}, 72{ }^{\circ} \mathrm{C}$ for $40 \mathrm{~s}$ ). Glyceraldehyde-3-phosphate dehydrogenase (GAPDH) was used as an internal control gene. The primer sequences: VEGF $\alpha$ (sense), 5'-GCCCTGAGTCAAGAGGACAG-3', and (antisense), 5'-CAGGCTCCTGATTCTTCCAG-3'; VEGFR2 (sense), 5'-TTTGGTAGCGGGATGAA-3', and (antisense), $5^{\prime}$-ATG GGATTGGTGAGGATGA-3'; eNOS (sense), 5'-TGACCCTCACC GATACAACA-3', and (antisense), $5^{\prime}$-CTGGCCTTCTGCTCATTTT C-3'; SDF- $1 \alpha$ (sense), $5^{\prime}$-TGTGCATTGACCCGAAATTA-3', and (antisense), 5'-CTTTGTGCTGGCAAATCTCAA-3', GAPDH (sense), 5'-AGACAGCCGCATCTTCTTGT-3', and (antisense), 5'-CTTGCCGTGGGTAGAGTCAT -3' were used. After validation of amplification efficiencies of target genes and the internal control gene, quantification of target gene expression was calculated using a $2^{-\Delta \Delta C T}$ method.

\section{Western Blot Analysis}

Lung tissue samples were analyzed for VEGF, VEGFR2, and eNOS protein expression using western blot analysis. Briefly, the frozen right lower lobes of rats were minced and homogenized in RIPA buffer containing a protease inhibitor cocktail. Fifty micrograms of total protein were separated on $10 \%$ SDS-polyacrylamide gels, and then electrophoretically transferred to a nitrocellulose membrane. Blots were blocked for $2 \mathrm{~h}$ in $10 \%$ nonfat dry milk in Tris Buffered Saline with $0.1 \%$ Tween 20 at room temperature. The membranes were incubated with rabbit polyclonal VEGF antibody (1:500, Abcam), rabbit polyclonal VEGFR2 antibody $(1: 1,000$, Abcam), and rabbit polyclonal eNOS antibody (1:1,000, Abcam) at room temperature overnight at $4{ }^{\circ} \mathrm{C}$. After washing, membranes were incubated with horseradish-peroxidase-conjugated anti-rabbit IgG (1:3,000 dilutions, Abgent, San Diego, CA) and horseradish-peroxidase-conjugated GAPDH (Kangchen, Shanghai,
China). The immunoreactivity was visualized using enhanced chemiluminescence.

\section{NO Assay}

NO production of the right vice lobe was evaluated by measuring the total $\mathrm{NO}^{-} / \mathrm{NO}^{-}$produced using the Griess reaction (Jiancheng Bioengineering Institute, Nanjing, China).

\section{Statistical Analysis}

All the continuous data were presented as means and SD. Continuous parametric variables were subjected to ANOVA for differences among groups, followed by post hoc Bonferroni testing for between-group differences. Nonparametric variables were subjected to KruskalWallis testing for differences among groups, followed by WilcoxonMann-Whitney test for between-group differences. $P$ values less than 0.05 were regarded as statistically significant.

\section{STATEMENT OF FINANCIAL SUPPORT}

This study was supported by the National Natural Science Foundation of China (No. 81070517, and 81270727).

Disclosure: We declare no confliction of interests regarding the work and paper contents. The study was not supported by any commercial entities, and has not been published elsewhere.

\section{REFERENCES}

1. Coalson JJ. Pathology of new bronchopulmonary dysplasia. Semin Neonatol 2003;8:73-81.

2. Abman SH. Bronchopulmonary dysplasia: "a vascular hypothesis". Am J Respir Crit Care Med 2001;164(10 Pt 1):1755-6.

3. Stenmark KR, Abman SH. Lung vascular development: implications for the pathogenesis of bronchopulmonary dysplasia. Annu Rev Physiol 2005;67:623-61.

4. Warner BB, Stuart LA, Papes RA, Wispé JR. Functional and pathological effects of prolonged hyperoxia in neonatal mice. Am J Physiol 1998;275(1 Pt 1):L110-7.

5. Rafat N, Tönshoff B, Bierhaus A, Beck GC. Endothelial progenitor cells in regeneration after acute lung injury: do they play a role? Am J Respir Cell Mol Biol 2013;48:399-405.

6. Borghesi A, Massa M, Campanelli R, et al. Circulating endothelial progenitor cells in preterm infants with bronchopulmonary dysplasia. Am J Respir Crit Care Med 2009;180:540-6.

7. Baker CD, Balasubramaniam V, Mourani PM, et al. Cord blood angiogenic progenitor cells are decreased in bronchopulmonary dysplasia. Eur Respir J 2012;40:1516-22.

8. Balasubramaniam V, Ingram DA. Endothelial progenitors in the risk of developing bronchopulmonary dysplasia: can we include endothelial progenitor cells in BPD risk assessment? Am J Respir Crit Care Med 2009;180:488-90.

9. Balasubramaniam V, Ryan SL, Seedorf GJ, et al. Bone marrow-derived angiogenic cells restore lung alveolar and vascular structure after neonatal hyperoxia in infant mice. Am J Physiol Lung Cell Mol Physiol 2010;298:L315-23.

10. Grisafi D, Pozzobon M, Dedja A, et al. Human amniotic fluid stem cells protect rat lungs exposed to moderate hyperoxia. Pediatr Pulmonol 2013;48:1070-80.

11. Kang WJ, Kang HJ, Kim HS, Chung JK, Lee MC, Lee DS. Tissue distribution of 18F-FDG-labeled peripheral hematopoietic stem cells after intracoronary administration in patients with myocardial infarction. J Nucl Med 2006;47:1295-301.

12. Templin C, Kotlarz D, Marquart F, et al. Transcoronary delivery of bone marrow cells to the infarcted murine myocardium: feasibility, cellular kinetics, and improvement in cardiac function. Basic Res Cardiol 2006;101:301-10.

13. Balasubramaniam V, Maxey AM, Morgan DB, Markham NE, Abman SH. Inhaled NO restores lung structure in eNOS-deficient mice recovering from neonatal hypoxia. Am J Physiol Lung Cell Mol Physiol 2006;291: L119-27. 
14. McCurnin DC, Pierce RA, Chang LY, et al. Inhaled NO improves early pulmonary function and modifies lung growth and elastin deposition in a baboon model of neonatal chronic lung disease. Am J Physiol Lung Cell Mol Physiol 2005;288:L450-9.

15. Donohue PK, Gilmore MM, Cristofalo E, et al. Inhaled nitric oxide in preterm infants: a systematic review. Pediatrics 2011;127:e414-22.

16. Vadivel A, Aschner JL, Rey-Parra GJ, et al. L-citrulline attenuates arrested alveolar growth and pulmonary hypertension in oxygen-induced lung injury in newborn rats. Pediatr Res 2010;68:519-25.

17. Grisafi D, Tassone E, Dedja A, et al. L-citrulline prevents alveolar and vascular derangement in a rat model of moderate hyperoxia-induced lung injury. Lung 2012;190:419-30.

18. Zhang R, Wang $L$, Zhang $L$, et al. Nitric oxide enhances angiogenesis via the synthesis of vascular endothelial growth factor and cGMP after stroke in the rat. Circ Res 2003;92:308-13.

19. Ekekezie II, Thibeault DW, Rezaiekhaligh MH, et al. Endostatin and vascular endothelial cell growth factor (VEGF) in piglet lungs: effect of inhaled nitric oxide and hyperoxia. Pediatr Res 2003;53:440-6.

20. Aicher A, Heeschen C, Mildner-Rihm C, et al. Essential role of endothelial nitric oxide synthase for mobilization of stem and progenitor cells. Nat Med 2003;9:1370-6.

21. Hristov M, Weber C. Endothelial progenitor cells: characterization, pathophysiology, and possible clinical relevance. J Cell Mol Med 2004;8:498-508.

22. Qi Y, Jiang Q, Chen C, Cao Y, Qian L. Circulating endothelial progenitor cells decrease in infants with bronchopulmonary dysplasia and increase after inhaled nitric oxide. PLoS One 2013;8:e79060.

23. Qi Y, Qian L, Sun B, Liu L, Wu P, Sun L. Inhaled NO contributes to lung repair in piglets with acute respiratory distress syndrome via increasing circulating endothelial progenitor cells. Plos One 2012;7:e33859.

24. Bo CJ, Chen B, Jia RP, et al. Effects of ischemic preconditioning in the late phase on homing of endothelial progenitor cells in renal ischemia/reperfusion injury. Transplant Proc 2013;45:511-6.

25. Asahara T, Murohara T, Sullivan A, et al. Isolation of putative progenitor endothelial cells for angiogenesis. Science 1997;275:964-7.

26. Yang J, Kamei N, Alev C, et al. CD34+ cells represent highly functional endothelial progenitor cells in murine bone marrow. Plos One 2011;6:e20219.

27. Kim CF, Jackson EL, Woolfenden AE, et al. Identification of bronchioalveolar stem cells in normal lung and lung cancer. Cell 2005;121:823-35.

28. Balasubramaniam V, Mervis CF, Maxey AM, Markham NE, Abman SH. Hyperoxia reduces bone marrow, circulating, and lung endothelial progenitor cells in the developing lung: implications for the pathogenesis of bronchopulmonary dysplasia. Am J Physiol Lung Cell Mol Physiol 2007;292:L1073-84.

29. Irwin D, Helm K, Campbell N, et al. Neonatal lung side population cells demonstrate endothelial potential and are altered in response to hyperoxia-induced lung simplification. Am J Physiol Lung Cell Mol Physiol 2007;293:L941-51.

30. Cao L, Qian LL, Zhu YR, Guo CB, Gong XH, Sun B. Regulation of activity of nuclear factor-kappaB and activator protein-1 by nitric oxide, surfactant and glucocorticoids in alveolar macrophages from piglets with acute lung injury. Acta Pharmacol Sin 2003;24:1316-23.

31. Bloomfield GL, Holloway S, Ridings PC, et al. Pretreatment with inhaled nitric oxide inhibits neutrophil migration and oxidative activity resulting in attenuated sepsis-induced acute lung injury. Crit Care Med 1997;25: 584-93.

32. Bloomfield GL, Sweeney LB, Fisher BJ, et al. Delayed administration of inhaled nitric oxide preserves alveolar-capillary membrane integrity in porcine gram-negative sepsis. Arch Surg 1997;132:65-75.

33. Ródenas J, Mitjavila MT, Carbonell T. Nitric oxide inhibits superoxide production by inflammatory polymorphonuclear leukocytes. Am J Physiol 1998;274(3 Pt 1):C827-30.

34. Qiao W, Niu L, Liu Z, Qiao T, Liu C. Endothelial nitric oxide synthase as a marker for human endothelial progenitor cells. Tohoku J Exp Med 2010;221:19-27.

35. Yin T, Ma X, Zhao L, Cheng K, Wang H. Angiotensin II promotes NO production, inhibits apoptosis and enhances adhesion potential of bone marrow-derived endothelial progenitor cells. Cell Res 2008;18:792-9.

36. Gallagher KA, Liu ZJ, Xiao M, et al. Diabetic impairments in NO-mediated endothelial progenitor cell mobilization and homing are reversed by hyperoxia and SDF-1 alpha. J Clin Invest 2007;117:1249-59.

37. Abe H, Ishikawa W, Kushima $\mathrm{T}$, et al. Nitric oxide induces vascular endothelial growth factor expression in the rat placenta in vivo and in vitro. Biosci Biotechnol Biochem 2013;77:971-6.

38. Valença SS, Rueff-Barroso CR, Pimenta WA, et al. L-NAME and L-arginine differentially ameliorate cigarette smoke-induced emphysema in mice. Pulm Pharmacol Ther 2011;24:587-94.

39. Ding BS, Nolan DJ, Guo P, et al. Endothelial-derived angiocrine signals induce and sustain regenerative lung alveolarization. Cell 2011;147: $539-53$.

40. Cooney TP, Thurlbeck WM. The radial alveolar count method of Emery and Mithal: a reappraisal 2-intrauterine and early postnatal lung growth. Thorax 1982;37:580-3. 